\title{
La construcción de procesos de identidad en las comunidades educativas, el caso del Colegio de Bachilleres del Estado de Chihuahua
}

\author{
Construction of identity processes in educational \\ communities, the case of the Colegio de Bachilleres of \\ the state of Chihuahua
}

\author{
Marilyn Georgia Salcido Sáenz* \\ Jesús Adolfo Trujillo Holguín**
}

\begin{abstract}
* Estudiante del Doctorado en Educación, Artes y Humanidades de la Universidad Autónoma de Chihuahua (México). Trabaja en la línea de investigación de Historia e Historiografía de la Educación. Cuenta con una maestría en educación superior y es licenciatura en ingeniería en sistemas computacionales en software por la Universidad Autónoma de Chihuahua. Actualmente se desempeña como docente del Colegio de Bachilleres del Estado de Chihuahua (COBACH) y realiza sus estudios en un programa que pertenece al Padrón Nacional de Posgrados de Calidad (PNPC), del Consejo Nacional de Ciencia y Tecnología (Conacyt). Correo electrónico georgiasalcidog9@gmail.com

(iD https://orcid.org/o000-0003-I09I-989X
\end{abstract}

*: Profesor-Investigador de la Universidad Autónoma de Chihuahua (México). Es doctor en educación por la Universidad Autónoma de Chihuahua y Maestro en Educación por la Universidad Pedagógica Nacional. Entre sus publicaciones recientes están: La educación en Ranchería Juárez, Chihuahua, I932-2018 (2019) y Debate legislativo y educación. El artículo tercero a cien años de la Constitución Política de 1917 (coord.) (20I8). Cuenta con reconocimiento al perfil Prodep y del Sistema Nacional de Investigadores Nivel I. Es socio del Consejo Mexicano de Investigación Educativa, de la Red de Investigadores Educativos Chihuahua y presidente de la Sociedad Mexicana de Historia de la Educación. Entre sus temas de interés están la historia e historiografía de la educación y formación de maestros. Correo electrónicojatrujillo@uach.mx

(D) https://orcid.org/oooo-0002-6738-1878

Historial editorial

Recibido: 24-julio-2019

Aceptado: 13-diciembre-2019

Publicado: 31-enero-2020

ISSN-e: 2594-2956 
La construcción de procesos de identidad en las comunidades educativas, el caso del Colegio de Bachilleres del Estado de Chihuahua

\section{Resumen}

El presente trabajo realiza un primer acercamiento a la identidad escolar de los alumnos del Colegio de Bachilleres del Estado de Chihuahua, tomando como referencia los conceptos que utiliza Mandoki (2006) sobre las matrices social y escolar y la manera en que influyen en la construcción de la identidad individual y colectiva de los sujetos. Las instituciones educativas se conciben como espacios significativos para la convivencia de los jóvenes, quienes están supeditados a reglamentos, códigos de conducta, apariencia, orden y disciplina; que permean en la formación de la identidad personal y escolar. Para estructurar el trabajo se recurre al análisis teórico del concepto de identidad, con el propósito de identificar cómo se construye en los actores del Colegio de Bachilleres durante los primeros años de la década de 1970, periodo que corresponde con su etapa de inicio. Las aportaciones teóricas se contrastan con la información que se obtuvo de fuentes orales, correspondientes a testimonios de actores que estuvieron como maestros y estudiantes en esa década. Entre los principales hallazgos destaca la identificación de contrastes en la formación de los jóvenes durante el periodo de funcionamiento de la Escuela Preparatoria de la Universidad Autónoma de Chihuahua y el viraje que hubo a partir de su clausura y del consiguiente nacimiento del Colegio de Bachilleres de Chihuahua en 1973.

244 Palabras Clave: Construcción de la identidad, Identidad, Contextos, Escuelas. 
Construction of identity processes in educational communities, the case of the Colegio de Bachilleres of the state of Chihuahua

\begin{abstract}
The present work attempts a first approach to the school identity of the students of the Colegio de Bachilleres del Estado de Chihuahua, a Chihuahua-based public high school system, based on Mandoki's (2006) concepts regarding social and school institutions and the way they affect the construction of the individual and collective identities of the subjects. Educational institutions are perceived as important spaces for the coexistence of teenagers, who are subject to regulations, codes of conduct, dressing codes, order and discipline, which permeate in the formation of personal and school identities. In order to structure this work, the theoretical analysis of the concept of identity is used, with the aim of identifying how it is built within the attendees of the Colegio de Bachilleres at the start of the I970s, a period that has been established as its starting point. The theoretical contributions are contrasted with the information obtained from oral sources obtained from testimonies of attendees who were either teachers or students during that decade. Among our main findings, contrast has been identified between the training of young people during the period of operation of the Universidad Autonoma de Chihuahua's high school and the change that took place since it was closed, as well as the subsequent creation of the Colegio de Bachilleres in 1973.
\end{abstract}

Keywords: Identity construction, Identity, Contexts, Schools 
La construction des processus identitaires dans les communautés éducatives, le cas du Collège des Licenciés de l'Etat de Chihuahua

\section{Résumé}

Le présent travail fait une première approche de l'identité scolaire des étudiants du Collège de Chihuahua, en prenant comme référence les concepts que Mandoki (2006) utilise sur les matrices sociales et scolaires et la manière dont ils influencent la construction de l'identité individuelle, et collectife des sujets. Les établissements d'enseignement sont conçus comme des espaces importants pour la coexistence des jeunes, soumis à des réglementations, codes de conduite, apparence, ordre et discipline, qui imprègnent la formación de l'identité personnelle et scolaire. Afin de structurer l' oeuvre, l' analyse théorique du concept d'identité est utilisée, dans le but d'identifier comment il se construit sur les acteurs du Collège du Baccalauréat au cours des premières années des années I970, période qui correspond á sa phase initiale. Les contributions théoriques contrastent avec les informations obtenues de sources orales, correspondant aux témoignages d' acteurs qui étaient proffesseurs qui étaient preofesseurs et étudiants au cours de cette décennie. Parmi les principales contrastations, l'identification de contrastes dans la formación des junes pendant la période de l' Ècole préparatorie de l'Université Autonome de Chihuahua et le changement inervenu depuis sa fermeture et la naissance de subséquente de l'École de Licence de Chihuahua en 1973.

Mots-clés: Construction identitaire, Contextes, Écoles, Identité. 
Budowa procesu tożsamości $w$ oswiacie, przypadek liceow ogolnoksztalcacych w stanu Chihuahua

\section{Streszczenie}

Zadaniem aktykulu jest zbadanie procesu tozsamosci $\mathrm{w}$ oswiacie na przykladzie liceow ogolnoksztalcacych w stanie Chihuahua, Punktem wyjscia jest praca Mandokiego (2006), w ktorej autor koncentruje sie na matryce szkolnej i jak wpływaja ona na budowanie indywidualnej i zbiorowej tożsamości uczestników. Instytucje edukacyjne są rozumiane jako przestrzenie o waznym znaczeniu dla młodych ludzi, którzy podlegają przepisom, kodeksom postępowania, wyglądowi, porządkowi i dyscyplinie; w zależności od kształtowania tożsamości osobistej i szkolnej. Do uporządkowania pracy stosuje się teoretyczną analizę pojęcia tożsamości, w celu określenia, w jaki sposób jbyla ona budowana $w$ liceach na poczatku lat 70-tych. Teoria kontrastuje $\mathrm{z}$ informacjami uzyskanymi ze źródeł ustnych, przede wszystkich od osob, ktore były nauczycielami i uczniami w tej dekadzie. Wynikim pracy jest identyfikacja kontrastów w szkoleniu młodzieży w pierwszym okresie funkcjonowania szkoły jako konsekwencji zamkniecia jedynego liceum w miescie, ktorego przyczyna bylo uczestnicwto w strajku przeciwko rzadowi w 1973.

Slowa kluczowe: Tozsamosc, konstrukcja tozsamosci, kontekst, oswiata 


\section{Introducción}

La construcción de la identidad de los sujetos es un proceso complejo que se ve influenciado por diferentes células de la sociedad: familia, escuela, religión, amigos, entre otras; las cuales influyen desde diferentes ópticas, para así crear cada una sus propias representaciones en el individuo. Particularmente, para el desarrollo de este artículo se retoma la importancia de la escuela en la conformación de la identidad de los sujetos, considerando la naturaleza de las comunidades educativas desde su entorno, sus procesos formativos, constructivos y dialógicos. Se asume que aun y cuando los sujetos tienen la libertad para formar su identidad como elección personal, también se ven influenciados por los contextos en que se ven inmersos y por los grupos a los que pertenecen.

Las escuelas como entes formativos de la sociedad desempeñan un papel significativo en la configuración identitaria de los individuos, debido a la socialización que se genera en estos espacios y a la subordinación de los sujetos a los ideales del centro educativo de pertenencia. En el mismo sentido, la confrontación y la interrelación que continuamente se suscita entre pares, docentes y otros intermediarios, favorecen la construcción de la identidad a través de la convivencia, bajo esquemas normativos que median las relaciones, ejerciendo influencia en los juicios propios y en la toma de decisiones.

El abordaje principal de este trabajo se centra en un acercamiento a la construcción de la identidad de la comunidad escolar del Colegio de Bachilleres del Estado de Chihuahua (COBACH) en sus primeros años de funcionamiento, la cual remite a escenarios sociales, culturales y políticos que caracterizaron la década de 1970. Metodológicamente se trata de una investigación histórica que se nutre de los testimonios orales obtenidos a través de la técnica de entrevistas semiestructuradas, realizadas con quienes estuvieron implicadas directamente en los hechos históricos. Asimismo, el análisis teórico se apoya en documentos y trabajos que enmarcaron el inicio de esta institución, se 
toman conceptos que ayudan a la compresión de la construcción de la identidad escolar y aspectos de la configuración identitaria que toma el Colegio dentro de sus códigos formativos y académicos.

El desarrollo de este trabajo se torna significativo debido a que se analizan los rasgos o características más distintivas de una de las instituciones más representativas del nivel medio superior del estado de Chihuahua, la cual surge en una época agitada y controversial dentro de la historia de la educación de nuestro país. Se ponen de manifiesto las relaciones que existen dentro de los códigos normativos de la escuela, su estructura institucional y los acontecimientos que le anteceden a su fundación, como son el movimiento estudiantil popular de 1968 y sus estragos para la educación en México, particularmente para la ciudad de Chihuahua, y los enfoques de la política educativa 1970-I976 del presidente Luis Echeverría Álvarez.

\section{Breves antecedentes}

El Colegio de Bachilleres del Estado de Chihuahua fue creado por decreto presidencial el i9 de septiembre de 1973, durante el sexenio presidencial del Lic. Luis Echeverría Álvarez. Sus antecedentes se encuentran en una iniciativa nacional que buscaba terminar con las escuelas preparatorias universitarias que brindaba la Educación Media Superior (EMS) en ese momento. La razón obedecía a diferentes problemáticas vinculadas con los movimientos estudiantiles que se suscitaron alrededor del país en los últimos años de la década de 1960 y primeros años de la de 1970; entre las que se encontraban la demandaba de espacios educativos para los jóvenes en edad de cursar el bachillerato.

En ese periodo existía una gran diversidad en los planes de estudio de la EMS, lo cual dificultaba la movilidad de los estudiantes. También, por la misma naturaleza de la educación universitaria, se concedía al estudiante de preparatoria todos los derechos y garantías de un universitario, lo que desencadenaba otro tipo de contrariedades, pues 
el nivel medio superior no se concebía como un fin, sino como un puente entre la educación secundaria y la superior. Por ello el gobierno federal, a través de la Asociación Nacional de Universidades e Instituciones de Educación Superior (ANUIES), se dio a la tarea de mediar la situación y dar solución a estos conflictos, los cuales fueron tratados en diversas reuniones (Salcido, Trujillo y Hernández, 20I8).

Sin embargo, no puede ignorarse el hecho de que también fue una medida para sosegar el descontento que existía en el país por el movimiento estudiantil de I968. Esta pugna acarreó nuevos conflictos que se propagaron por toda la República y encontraron como venero de la disputa a las escuelas preparatorias universitarias, donde sus estudiantes gozaban de privilegios concedidos bajo la autonomía de las universidades y constituían el grueso de la matrícula en dichas instituciones. Ontiveros y Pérez (2018), mencionan que “[...] el movimiento estudiantil chihuahuense se vinculó a los conflictos sociales del Estado, como el Comité de Defensa Popular (CDP) [...] se inspiró en las tendencias de orden nacional como el movimiento estudiantil del 68" (p. 56). Señalan que los estudiantes de la Escuela Preparatoria de la Universidad Autónoma de Chihuahua simpatizaron con "[...] la clase media en un principio, demandaban una transformación reformista, pero conforme se fueron desarrollando los procesos políticos en el contexto social, fueron radicalizándose e identificándose cada vez más con los sectores populares, campesinos y obreros" (Ontiveros y Pérez, 20I8, p. 58).

Particularmente para la ciudad de Chihuahua, la Escuela Preparatoria de la Universidad siempre se distinguió por su esencia humanista y figuraba como un espacio para la discusión de ideas y el debate de opiniones según las diferentes ideologías que manifestaban los jóvenes. Esta característica ocasionó que fuera un foco de conflicto para el gobierno, pues era relativamente fácil envolver a los jóvenes en manifestaciones y huelgas que -con el paso del tiempo- llevaron al cierre de dicha institución y al posterior inicio del Colegio de Bachilleres (A. Rico, comunicación personal, 20 de febrero, 20I8). 
El cierre de la Escuela Preparatoria en 1973 se debió a una huelga que ocasionó daños en diferentes espacios de la institución. Aunque anteriormente se habían suscitado otros conflictos, este último fue el que dio fin a la insigne escuela. Bajo estas premisas, un grupo de personas, entre quienes figuraba el que posteriormente sería el primer director sectorial del COBACH -Lic. Oscar Ornelas Küchle- pidieron el adelanto del proyecto del Colegio de Bachilleres en la ciudad de Chihuahua, mismo que estaba programado para iniciar sus actividades en la Ciudad de México (Villegas, 1992). La finalidad primordial era crear un nuevo sistema de nivel medio superior emancipado de la educación universitaria, que diera respuesta a la demanda de espacios educativos y que acabara de tajo con el problema en la Escuela Preparatoria (G. Cendón, comunicación personal, I4 de noviembre, 20I8).

El COBACH inició labores en la ciudad de Chihuahua en septiembre de 1973, contando con tres planteles que funcionaban en los turnos matutino y vespertino, a excepción del plantel 2, que ofrecía el turno nocturno para atender a la población trabajadora que deseara concluir sus estudios preparatorios.

\section{La exploración de la identidad escolar en la construcción de una nueva sociedad}

El Colegio de Bachilleres debía responder a las problemáticas de la época y al mismo tiempo obedecía a los planteamientos en los que se centró la política educativa del sexenio del presidente Luis Echeverría Álvarez (1970-1976). Pablo Latapí menciona que la educación en ese periodo se orientó hacia los siguientes enfoques:

Como bien en sí mismo: es decir, como beneficio social que enriquece la cultura de amplios sectores de la población, asegura la unidad nacional y contribuye al desarrollo económico del país; como medio de legitimación ideológica del sistema político mexicano y del propio gobierno; como oferta de un camino de 
ascenso social, principalmente para las clases medias; como dádiva política en la negociación de apoyos, particularmente de las clases más desprotegidas suburbanas y rurales, para las cuales la educación es el beneficio social más inmediato y deseable (Latapí, I980, pp. 60-6I).

Por tanto, la educación dentro de la gestión echeverrista tenía la encomienda de generar una transformación social, considerándose "prudente y oportuno la creación de un sistema que diera una nueva estructura y ordenamiento al nivel medio superior" (Salcido, Trujillo y Pérez, 20I9, p. 3). Además, la creación del Colegio atendía a la estrategia de innovación educativa que "se basa en instituciones nuevas, creadas al lado del sistema convencional, con la intención de que lleguen a desplazarlo" (Latapí, I980, p. 228). El COBACH siendo parte de estas nuevas instituciones, contribuiría a una iniciativa de renovación de suma importancia para la historia de la educación. Por consiguiente, "la estrategia paralela a base de instituciones innovadoras cuidadosamente diseñadas" (Latapí, I980, p. 229) podría generar un cambio verdadero hacia una transformación gradual y estructural, para llevar "la evolución de la sociedad hacia formas más justas de convivencia, y que la educación tiene contribuciones significativas que hacer a este proceso" (Latapí, I980, p. 230).

En las transformaciones propuestas por la política pública federal se toma a la escuela como una vía de renovación de la sociedad, considerando que el individuo a lo largo de la vida va siendo participe de diferentes matrices sociales. Entiéndase como matriz el planteamiento que hace Mandoki:

[...] las matrices del latín 'mater', son literal y metafóricamente los lugares donde se gesta y se desarrolla la identidad [...] son focos de irradiación y productos de identidades. Desde diversas matrices, el sujeto se contagia de modos particulares de ver y sentir el mundo y la vida" (2006, p. 7I y 8I). 
Desde ese enfoque teórico, una de las matrices que resulta importante para el sujeto es la escolar, pues un individuo promedio pasa casi un tercio de su vida en las escuelas y -aunque las matrices son colectivasdejan una particular marca en cada persona, al tiempo que adquieren una identidad institucional que los hace pertenecer a ese organismo. Los sujetos son capaces de identificarse en un tiempo y espacio con quienes compartieron ese periodo y aunque la manifestación de cada persona es de carácter y personalidad individual, en conjunto logran mantener un estilo que adquieren de ese contexto matricial.

De acuerdo con la idea anterior, los estudiantes y egresados del Colegio de Bachilleres eran instruidos y formados bajo ciertos códigos y reglamentos con los cuales se iban fijando comportamientos que eran comunes entre los individuos, debido a la oficialidad del organismo y a las convicciones que se inculcaban, por medio de la educación formalizada (Mandoki, 2006). Según el mismo autor, la importancia y la trascendencia que la matriz escolar tiene para la vida de los sujetos es de altísima primacía, debido a que está subyugada al Estado y a sus intereses, tal como fue el caso del Colegio de Bachilleres, pues al momento de su creación fue evidente la intención que tenía el gobierno en la formación de los jóvenes, la disciplina, el orden y la imagen. Estas eran cuestiones fuera de negociación y tenían que acatarse como lo señalaba la norma.

En el Reglamento interior de convivencia escolar para el alumnado del Colegio de Bachilleres del Estado de Chihuahua, se destina el Capítulo III para especificar las obligaciones relacionadas con orden, disciplina e imagen de los estudiantes y que se detallan en el artículo i5 de la siguiente manera:

[...] los y las alumnos/as del COBACH tendrán las obligaciones inherentes a su condición académica y además las que a continuación se indican: [...] X. Conservar y mantener el prestigio institucional, mediante una conducta acorde a su condición de alumno/a del COBACH, dentro y fuera de sus 
instalaciones y/o Instancia educativa [...] XI. Portar el uniforme debidamente aseado y conforme a los lineamientos establecidos en cada una de las modalidades [...] XX. Las actitudes que observen las alumnas/alumnos dentro y fuera del plantel deberán ser positivas: de respeto, responsabilidad, orden y decoro; máxime si portan algún uniforme representando a la institución en alguna actividad cívica, social o deportiva. (Art. 55 de la Ley Orgánica) [...] XXI. Toda acción o manifestación que atente contra el orden, la moral y las buenas costumbres podrá ser atendida por [todo el personal académico y administrativo del plantel] (COBACH, 2OI7, pp. IO-I2).

Bajo estas premisas, estaba de manifiesto la importancia y preeminencia que tenía el orden y la disciplina para la institución. Sus alumnos debían apegarse a las normas desde el momento de la inscripción, ya que eran parte de su identidad y un requisito para formar parte de esa comunidad educativa. Asimismo, la institución dio una fuerte significación a la imagen de sus estudiantes, lo cual también se ve regulado en su Ley Orgánica y en el reglamento de convivencia para los alumnos.

Mandoki (2006) menciona que "la proxémica escópica dominante en la matriz escolar impone a los alumnos el estricto uniforme para establecer una distancia larga entre el hogar y la escuela, y respecto a una escuela y otra [...], una proxémica de clase" (p. 152). En este sentido, proxémica escópica "se refiere a la puesta en vista de distancias largas y cortas por medio de artefactos o usos del espacio" (Mandoki, 2006, p. 48), por lo que, el uso de un atuendo en las escuelas marca un estilo, una época, un precio y una jerarquía. Propiamente el Colegio no impuso de 254 principio el uso del uniforme debido a que era una época de transición, en donde gradualmente se llegó a la institucionalización y reglamentación del uso del atuendo, pues en la antigua Escuela Preparatoria no era requerido el uso de una indumentaria especifica ni regulada. Sin embargo, el COBACH si tenía un código que regulaba la 
forma de vestir (G. Cendón, comunicación personal, I4 de noviembre, 2018 y R. García, comunicación personal, I4 de marzo, 20I8).

Existen cuatro conceptos que están relacionados en el intercambio matricial de experiencias en las instituciones sociales: los sintagmas, los paradigmas, las matrices y las instituciones. Se conceptualizan de la siguiente manera:

[...] las matrices son colectivas, la combinación de sus huellas paradigmáticas es única en cada individuo [...] el paradigma es un marco de referencia en función al cual un enunciado o sintagma significa por diferenciación y simboliza por asociación [...] la institución es solo un segmento de las matrices en el que se han coagulado prácticas al oficializarse por normas explícitas impersonales como [...] un reglamento escolar [...] la institución fija comportamientos, mientras que la matriz los engendra continuamente (Mandoki, 2006, pp. 77-8I).

De acuerdo con el autor, la diferencia entre una matriz y una institución está en que la última es un estrato de la primera. Para nuestro caso, el Colegio de Bachilleres es una institución que forma parte de la matriz escolar de la Educación Media Superior (EMS), de manera que las instituciones en su organización imponen esquemas de comportamiento que están supeditados a la disciplina, el orden, el desarrollo de habilidades y la integración de una calificación.

El individuo, como un ser holísticamente social, se ve comprometido a interactuar de diferentes maneras con la realidad. Su cotidianidad lleva en sí misma procesos de objetivación, institucionalización y legitimación que están ligados directamente al desarrollo de la identidad, la cual se verá influenciada desde la subjetividad de cada persona, de quienes le rodean y de los lugares y espacios en los que se ve inmersa. Los diferentes roles que el sujeto adquiere durante su vida, van tomando parte en la construcción de su identidad individual y grupal. Por lo tanto, el estudiante del COBACH -al pertenecer a esta 
institución- se vio inmerso en la oficialidad y se sujetó a la educación formalizada que se le inculcaba (Mandoki, 2006).

Mandoki (2008) menciona que el ser humano experimenta en su subjetividad tres facetas que lo diferencian de otros seres vivos: individualidad, identidad y rol. La individualidad toma parte en los procesos biológicos del sujeto, configurándolo como un ser único en el tiempo y espacio, que irá conformando su carácter en el desarrollo de todas sus etapas. Por otro lado, la identidad se constituye en los procesos sociales, es decir, permean las interacciones con los demás porque los sistemas sociales están hechos para que el individuo tenga que manifestarse en ellos de manera imperativa. La familia, la raza, la nacionalidad, la profesión, la religión y el contexto, son matrices sociales que modelan la identidad.

El individuo sí es participe en la configuración de su identidad y aunque esta se conceda a través de las estructuras sociales a las que pertenece, el sujeto es un participante activo en dicho proceso. La identidad adquiere un valor estético más que semiótico, puesto que la apreciación de los otros se traduce en una valoración de la personalidad en términos de belleza.

Considerando las anteriores premisas, el estudiante del COBACH era apreciado por la sociedad porque se distinguía según la institución de pertenencia. A esta le precedía el prestigio de calidad educativa heredado de la Escuela Preparatoria y reforzado por su imperativo reglamento (R. García, comunicación personal, I4 de marzo, 20I8). De ahí que la identidad tome una apreciación estética, interpretada por los semejantes a través de valoraciones positivas y negativas derivadas de sus propias interacciones en diferentes estructuras sociales y otorgadas por la realidad en la que se habita (Mandoki, 2008). Los estudiantes del $\mathrm{COBACH}$ eran personas capaces de acatar reglas; sin embargo, esto no solo radica en el hecho de formar buenos estudiantes, sino de seleccionar o admitir buenos alumnos. Raymundo García Quintana, 
siendo el orador en la ceremonia de graduación de la primera generación de egresados del $\mathrm{COBACH}$, menciona:

A nosotros de un solo tajo nos quitaron la ilusión de seguir pensando, pero nuestro objetivo era seguir estudiando, así que reunimos los requisitos, nos cortamos el pelo, hubo compañeros que no quisieron, pero no entraron, no se les permitió ingresar, el régimen era muy duro para poder entrar (comunicación personal, I4 de marzo, 20I8).

La identidad es otorgada mantiene un carácter personal y propio del sujeto, en el que las matrices sociales aportan en su configuración. Al mismo tiempo, el sujeto toma conciencia en la construcción de su identidad dándole la condición de especificidad propia y subjetiva. La última faceta de la subjetividad es el rol, el cual toma una naturaleza anónima y general, que mayormente se asume $\mathrm{o}$-mejor dicho- adquiere un carácter circunstancial, que se dará a través de las diferentes matrices a las cuales pertenece el sujeto (Mandoki, 2008).

El Colegio de Bachilleres se manifestaba entonces como una nueva matriz social y escolar. La institución, siendo un espacio para la convivencia y la formación de los jóvenes, se manifestaba como una matriz que trasmitía su forma particular de ser joven y ser estudiante, dando una homogenización mental al alumnado para ejercer dominio sobre ellos, sometiéndolos a los propósitos del sistema.

Bajo estas premisas, el Colegio de Bachilleres se convirtió en la primera opción para el ingreso a la EMS, debido a que ocupó el lugar que anteriormente tenía la Escuela Preparatoria. Era ineludible que sus valores e ideales permearán en la juventud chihuahuense y por ello se introducen en las generaciones de alumnos que han pasado por sus aulas desde 1973 hasta la fecha. Sus estudiantes se encuentran inmersos en un intercambio social concreto, donde existen negociaciones entre las diferentes identidades de los individuos que ahí concurren: docentes, directivos, administrativos y el resto de los jóvenes. 
Además, los diferentes estilos de vida y clases sociales de pertenencia tomaron parte en esta negociación, porque cabe decir que el Colegio de Bachilleres, aun siendo una escuela pública, logro introducirse en diferentes esferas sociales. Ello se debió -en primera instancia- a que era la única opción educativa de nivel medio superior que ofrecía el Estado y a su condición como sucesora de la Escuela Preparatoria, de la cual heredó cierto prestigio. Por estas razones el COBACH fue un espacio heterogéneo en clases sociales, que podía albergar alumnos de clase media-alta, media y baja en un mismo plantel. Esta característica hizo que el intercambio de experiencias en la matriz social y escolar fuera tan enriquecedora y al mismo tiempo de grandes contrastes.

\section{La disciplina como esquema constituyente de la educación formal en el COBACH}

Considerando los párrafos anteriores como una premisa para la configuración del esquema que caracterizó al COBACH durante sus primeros años, resalta la importancia de los procesos de objetivación, institucionalización y legitimación que ocurren en los espacios escolares para la conformación de la identidad. El COBACH tomó como eje la disciplina, de manera que le permitiera llevar a cabo todos sus procesos académicos y formativos. Dicho concepto se entiende como los "métodos que permiten el control minucioso de las operaciones del cuerpo, que garantizan la sujeción constante de sus fuerzas y les imponen una relación de docilidad-utilidad" (Foucault, 2002, p. I26).

De acuerdo a las circunstancias de inicio, la configuración institucional del COBACH se vio afectada por las coyunturas sociales y políticas que jugaron un papel crucial en la definición de la escala de control que se quería tener sobre los estudiantes. En la nueva configuración de la sociedad no era aceptable que existieran espacios para la generación de movimientos que agitaran el orden establecido.

El COBACH -a través de la disciplina- ejercía la clausura de sus espacios como una forma de delimitarse con lo exterior, es decir, que proponía- 
a través de su reglamento- una nueva forma de ser joven y de alejarse del bullicio de la época post '68. El acotamiento de los diferentes planteles resguardaba un nuevo tipo de control que la antigua Escuela Preparatoria no poseía. Desde su concepción se propuso segregar a los estudiantes en diferentes espacios y con ello se logró:

Anular los efectos de las distribuciones indecisas, la desaparición incontrolada de los individuos, su circulación difusa, su coagulación inutilizable y peligros [...] [y consiguió] establecer las presencias y las ausencias, de saber dónde y cómo encontrar a los individuos, instaurar las comunicaciones útiles, interrumpir las que no son, poder en cada instante vigilar la conducta de cada cual, apreciarla, sancionarla, medir las cualidades o los méritos (Foucault, 2002, p. I3I).

Asimismo, el COBACH utilizó los emplazamientos funcionales, que se definen como espacios creados con la finalidad de custodiar, fijándose una especie de filtro para mantener el orden y la armonía en las relaciones de pares. Esta función se llevaba a cabo mediante las prefecturas, las cuales se encargaban de hacer valer el reglamento y de cuidar la sana convivencia entre alumnos y maestros. Foucault (2002) menciona que "la primera de las grandes operaciones de la disciplina, es, pues, la constitución de cuadros vivos que transforman las multitudes confusas, inútiles o peligrosas en multiplicidades ordenadas" (p. 136). De esta manera, la exigencia del orden se manifestaba como una técnica de poder y dominación para los estudiantes, bajo esquemas de empleo del tiempo que definían ritmos de clase y requerimientos de buenas conductas, las cuales estaban aseguradas durante toda la jornada escolar. La imposición de diferentes métodos disciplinarios buscaba generar y reproducir un tiempo integralmente útil para la vida académica de los estudiantes, lo cual coadyuvaba a alcanzar el principio de no ociosidad.

El Colegio de Bachilleres inició con un esquema de escuela innovadora, debido a que incorporaba las recomendaciones de la ANUIES, 
relacionadas con la reforma de los planes de estudio de dos a tres años, inclinándose más hacia las ciencias duras y relegando el papel formativo de las ciencias sociales. Sin embargo, figuraba dentro de la institución un modelo evidente de autoridad y dominio en sus procesos formativos y académicos, a través del uso de métodos como la obediencia, uso de sanciones, pasividad y fuerte sujeción a un modelo de estudiante pulcro y correcto. Para el COBACH, el uso de estos procedimientos -específicamente la disciplina- no fue solo una forma de obstaculizar la insubordinación o deserción de los estudiantes, sino que se manifestaba como forma para que la institución permaneciera como un ente que acrecentara las habilidades y el rendimiento del alumnado. Al respecto, Raymundo García Quintana señala:

Eran muy estrictos, había normas muy fuertes de disciplina y de uniforme, las mujeres también tenían sus reglas, sin embargo, para ellas creo fue mejor el cambio no se permitían las minifaldas, en la preparatoria si, acá fueron más tradicionales (comunicación personal, I4 de marzo, 2018).

Los primeros modelos que se usaron para el Colegio de Bachilleres fueron imperativos por parte de los docentes. Sus procesos estaban orientados a la enseñanza de contenidos, la pedagogía predominante fue autoritaria y bajo un fuerte régimen de disciplina. La labor de los profesores durante los primeros años se orientó a enseñar, evaluar, precisar normas, controlar y vigilar. El COBACH adoptó como función social la de instruir a los estudiantes para llegar a un proceso de transformación de la sociedad mediante la práctica de actitudes y valores, los cuales se implicarían en la vida social del sujeto y de esta forma se iniciaba una renovación que comenzaría en la escuela y tendría un impacto directo en la ciudadanía.

El sistema que creó el COBACH para la adquisición, transmisión y uso del conocimiento se hizo posible a través de una plantilla docente que fuera competitiva y comprometida con la función social de la institución y que pudiera responder a las exigencias sociales de la 
época. Asimismo, el sistema de intercambio de conocimientos debía responder a las demandas del contexto y dotar a los estudiantes de las competencias básicas para asegurar el éxito en la educación superior.

El Colegio de Bachilleres nace en una época subversiva, por lo que los rasgos que lo definieran debían ser contrarios al periodo de caos que le antecede. En ese periodo -principios de la década de 1970- la educación se distinguió por dar un giro hacia el sector industrial, el cual estaba definido por criterios funcionalistas internacionales que dirigían a la juventud hacia términos como productividad, competitividad y optimización. El ámbito cultural y humanista de la educación se alejó de las aulas, con limitaciones muy marcadas (Cruz, 2002).

El proyecto educativo desarrollado en la gestión del presidente Luis Echeverría Álvarez consideraba "la formación de nuevas subjetividades" (González, 20I8, p. I08) como una vía para la reforma educativa. Por esa razón el COBACH debía apegarse a los criterios señalados, ya que emanaba del mismo proyecto educativo y requería lograr el cambio en los individuos, sus valores, su sistema de creencias y sus prácticas. Las instituciones estaban orientadas a formar individuos bajo "la definición de un nuevo sujeto: [...] el individuo en proceso de formación, que la escuela orienta, capacita y desarrolla en libertad" (González, 20I8, p. IIO).

El papel que desempeñaba el maestro dentro del Colegio era el de ejercer:

Un tipo específico de poder: ya no solo el guía que los acompaña en la adquisición de conocimientos, sino el maestro-gobernante, que trabaja sobre la conducta, los afectos, los impulsos, las acciones y los sentimientos de individuos que atraviesan un proceso crítico, un proceso de formación del carácter y la personalidad, también de preparación para la vida (González, 20I8, p. II4). 
La tarea educativa de la época fue el desarrollo de un sujeto conveniente para el sistema de gobierno. Se debía "homogeneizar mentalmente a los educandos para el control social" (Mandoki, 2006, p. I48). Es importante destacar -como se dijo antes- que, aunque el COBACH surgió en la ciudad de Chihuahua como respuesta a un problema político, desde principios del sexenio de Luis Echeverría se tenía planeado la diversificación de la EMS en el país, además de la creación de nuevas instituciones que brindaran espacios educativos a los jóvenes en edad de cursar el bachillerato. El Colegio de Bachilleres alineó todos sus estatutos para cumplir con los acuerdos y recomendaciones generadas en las asambleas convocadas por la ANUIES en I97I y 1972, por lo que su estructura académica se organizó con base a los lineamientos de la enseñanza media superior (Villegas, 1992).

Ahora bien, la intencionalidad en la política educativa del presidente Luis Echeverría fue poner de manifiesto un cambio en las estructuras mentales de los jóvenes. En varios discursos el mandatario resaltó que los problemas por los que atravesaba el país se debían principalmente al ámbito educativo y señaló que no era posible alcanzar el progreso económico y social, sin antes atender a la demanda de espacios que se requerían para cubrir las necesidades educativas y llegar a más sectores de la población (Núñez, 20Io). Evidentemente el discurso político comprendía el cambio de estructura mental y la expansión de la alfabetización a lo largo de la vida educativa. Por medio de la reforma se buscaba un cambio en todos los niveles del sector educativo y con ello la creación de nuevas instituciones que llegaran a los sectores marginados de la población, aquellos a quienes no les era posible acceder a la educación debido a su condición social. Sin embargo, pese

262 a que la iniciativa logró su cometido, no se puede dejar de lado que la intencionalidad fue siempre un cambio mental desde las entrañas, desde lo formativo, para que todas sus actividades se supeditaran a la disciplina, el orden y la imagen.

\section{Conclusiones}


Considerando la magnitud del Colegio de Bachilleres para el estado de Chihuahua, se puede observar que la institución logra identificarse como una matriz, tal como menciona Mandoki, por el hecho de que produce identidades, en las cuales se pueden advertir particularidades en el modo de comprender la vida. Hay algo en común para las personas que pasaron por el Colegio de Bachilleres y es la ideología que les inculcaron de manera tamizada, en relación a principios basados en la disciplina, orden y cuidado de la imagen; contrario a lo que ocurría en la Escuela Preparatoria, donde era obvia la ideología de izquierda y el espíritu contestatario y de debate que predominaba entre sus estudiantes.

Considerando las circunstancias en que nace el Colegio de Bachilleres y los cometidos que llevaba en sus inicios, a través de ellos se formaron miles de jóvenes -durante cuarenta y siete años- bajo banderas de conducta que no atentaran contra los reglamentos y códigos de la institución.

La subordinación es algo que se logró identificar en los sujetos que ocuparon los espacios educativos del Colegio de Bachilleres. Son notorias ciertas características en los estudiantes, pues la institución -al configurarse como una entidad formativa que sirve al Estado- tenía que cumplir reglamentos estrictos, mantener una imagen de decoro y pulcritud, y estas eran solo algunas de las subordinaciones que los estudiantes tenían que acatar para pertenecer y permanecer en esta institución.

Uno de los frutos de la configuración identitaria del Colegio de Bachilleres ha sido el desarrollo armónico de las facultades de los estudiantes para su crecimiento educativo, familiar y profesional. La formación de una actitud positiva y de respeto hacia la escuela ha propiciado estabilidad para el sector educativo chihuahuense. El COBACH logró mantener un equilibrio que regulara la generación de recursos humanos para el ingreso a la educación superior y/o al sector productivo de manera exitosa. Aún y con la rigidez del sistema, se puede 
decir que el Colegio de Bachilleres logró mantener la función social que se le encomendó, ya que dio orden a la EMS en el estado. Además, brindó espacios para atender la demanda educativa que se detonó en los primeros años de la década de 1970.

El Colegio de Bachilleres, desde sus planteamientos, estableció un puente de movilidad social para sus estudiantes, siendo el espacio escolar un medio para la preparación de jóvenes bajo esquemas que encajaran en la nueva era de la industrialización que vivió el país a partir de la década de los setenta. El acceso a la educación en sectores marginados de la población aseguró una mejor posición social y económica de las personas.

\section{Referencias}

Cendón Chávez, G. (20I8, I4 de noviembre). Entrevista personal. Chihuahua, México.

COBACH. [Colegio de Bachilleres del Estado de Chihuahua]. (2017). Reglamento interior de convivencia escolar para el alumnado del Colegio de Bachilleres del Estado de Chihuahua. Chihuahua, México: Colegio de Bachilleres del Estado de Chihuahua. Recuperado de http://www.cobachih.edu.mx/Portals/o/Documentos/Reglamen to\%20Definitivo\%2oFebrero\%202018.pdf?ver=2018-02-23090305-680

Cruz, M. (2002). Influencias ideológicas en las modificaciones al artículo tercero constitucional. En D. Piñera (Coord.), La educación superior en el proceso histórico de México: Cuestiones esenciales, prospectiva del siglo XXI (pp. 98-I2I). Mexicali, México: ANUIES.

Foucault, M. (2002). Vigilar y Castigar: nacimiento de la prisión. Buenos Aires, Argentina: Siglo XXI. 
García Quintana, R. (20I8, I4 de marzo). Entrevista personal. Chihuahua, México.

González, R. (20I8). La reforma educativa en México: 1970-I976. Espacio, tiempo y educación, 5(I), 95-II8. DOI: http://dx.doi.org/IO.I45I6/ete.2I4

Hernández Orozco, G., Pérez Piñón F.A., y Evangelista Márquez, J.L. (20I0). El Instituto Científico y Literario de Chihuahua I934-I954. Chihuahua, México: Universidad Autónoma de Chihuahua.

Latapí, P. (1980). Análisis de un sexenio de educación en México, 1970-1976. México: Editorial Nueva Imagen.

Mandoki, K. (2006). Prácticas estéticas e identidades sociales: prosaica dos. México: Siglo Veintiuno Editores.

Mandoki, K. (2008). Estética cotidiana y juegos de la cultura: prosaica I. México: Siglo Veintiuno Editores.

Núñez Jaramillo, E. (2010). Aportes de la reforma educativa del sexenio de Echeverría (I970-1976) a la Educación Media Superior. Orígenes de dos proyectos: Colegio de Bachilleres y Colegio de Ciencias y Humanidades (CCH). México: Universidad Nacional Autónoma de México.

Ontiveros Juárez, G., y Pérez Piñón, F.A. (2018). Balances y aproximaciones teóricas al movimiento estudiantil. Debates por la Historia, VI(I), 39-63. Recuperado de: https://vocero.uach.mx/index.php/debates-por-lahistoria/article/view/9/14

Rico Bovio, A. (2018, 20 de febrero). Entrevista personal. Chihuahua, México. 
Salcido Sáenz, M.G., Trujillo Holguín, J.A., y Hernández Orozco, G. (2018). La Educación Media Superior en Chihuahua I827-1973. Una aproximación a los antecedentes para la fundación del sistema COBACH. RECIE. Revista Electrónica Científica de Investigación Educativa, 4(I), 35-46. Recuperado de https://rediech.org/ojs/20I7/index.php/recie/article/view/292

Salcido Sáenz, M.G., Trujillo Holguín, J.A., y Pérez Piñón, F.A. (2019). Análisis microhistórico de un proceso de transformación social a partir de las instituciones educativas, el caso del Colegio de Bachilleres. Ponencia presentada en el XV Congreso Nacional de Investigación Educativa. Acapulco, México. Recuperado de http://www.comie.org.mx/congreso/memoriaelectronica/vi5/do c/2666.pdf

Villegas, J. (1992). Educación pública y bachillerato, la función social del Colegio de Bachilleres [Tesis de licenciatura no publicada]. Universidad Nacional Autónoma de México, México.

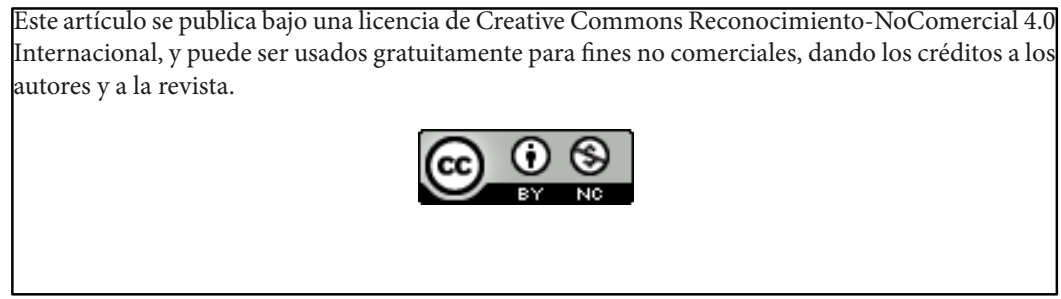

